\title{
Bioinformatics analysis reveals different gene expression patterns in chondrocytes from knee and finger joints: implications for the effect of mechanical stress in the osteoarthritic development
}

\section{Wei Mei}

Nanjing University of Chinese Medicine

Songjiang Yin

Nanjing University of Chinese Medicine

Xiaoqing Shi

Nanjing University of Chinese Medicine

Xiaochen Li

Nanjing University of Chinese Medicine

\section{Li Zhang}

Nanjing University of Chinese Medicine

Runlin Xing

Nanjing University of Chinese Medicine

Nongshan Zhang

Nanjing University of Chinese Medicine

\section{Peimin Wang}

Nanjing University of Chinese Medicine

Guicheng Huang ( $\nabla$ drhgc2020@163.com )

Nanjing University of Chinese Medicine

\section{Research}

Keywords: Mechanical Stress, Osteoarthritis, Differentially Expressed Genes, Bioinformatics Analysis

Posted Date: April 21st, 2020

DOl: https://doi.org/10.21203/rs.3.rs-22653/v1

License: (1) This work is licensed under a Creative Commons Attribution 4.0 International License. Read Full License 


\section{Abstract \\ Background}

Mechanical stress has been well known to be a significant risk factor for the onset and progression of osteoarthritis (OA). The objective of this study is to clarify the key genes and the signaling pathways which is associated with the effect of mechanical stress on OA development.

\section{Results}

A total of 213 Differentially expressed genes (DEGs) were identified in cultured chondrocytes from knee and finger joints, including 101 up- and 112 down-regulated genes, which brought an enrichment in positive or negative regulation of cell proliferation, positive regulation of cell division, positive regulation of apoptotic process and embryonic limb morphogenesis. Two comparison cohorts shared 122 overlapping genes including 49 up- and 73 down-regulated genes, which enriched in DNA replication, cell division, calcium ion binding and positive regulation of apoptotic process. Twelve hub overlapped genes including Cyclin dependent kinase 1 (CDK1), Kinesin family member 11 (KIF11), Mitotic checkpoint serine/threonine kinase (BUB1) and Mitotic arrest deficient 2 like 1 (MAD2L1) were defined and predicted miRNAs of the genes mainly enriched in Transforming growth factor beta (TGF- $\beta$ ) signaling pathway, Wnt signaling pathway and Mitogen activated kinase-like protein (MAPK) signaling pathway.

\section{Conclusions}

Mechanical stress shown multiple influences on chondrocytes, physiologically necessary for maintaining chondrocyte homeostasis and promoting cartilage development, pathologically help for cartilage destruction and OA development via boosting chondrocyte differentiation and hypertrophy and accelerating cell apoptosis.

\section{Introduction}

Chondrocytes, the resident cells in articular cartilage, maintain the matrix components under normal or low turnover conditions [1]. As mechanosensitive cells, chondrocytes, especially in a weight-bearing joint, could alter phenotype and function in perceiving and responding to mechanical stress [2]. Physiological dynamic loading exerts chondroprotective effect via initiating anti-catabolic and anti-inflammatory signals involved in chondrocyte homeostasis [3, 4]. Pathologically, mechanical stimulation contributes to the activation of catabolic processes by altering specific signal pathway $[5,6]$ and even involves in the induction of chondrocyte apoptosis [7]. Typically, when the chondrocyte homeostasis is disturbed by mechanical loading, articular cartilage destruction and degeneration occur, resulting in joint pain and dysfunction, clinically identified as osteoarthritis (OA) [8]. 
$\mathrm{OA}$ is the most prevalent joint disease and a leading source of chronic pain and disability in the world [9]. Knee OA accounts for more than $80 \%$ of the disease's total burden [10] and emerging evidence indicates that knee $O A$ is proximately caused by the breakdown of joint tissues from mechanical stress [11]. The knee joint, the most weight-bearing joint in the human body, is more susceptible to mechanical stress, especially in the medial compartment [12]. Excessive or repetitive mechanical stress in knee joint could cause progressive cartilage degeneration and subsequent development of the clinical OA [13].

Consequently, the concept of mechanical stress leading to damaged joint and OA development is widely supported by previous reports [14-15]. However, almost researchers merely focused on the OA-related individual gene, which fail to comprehensively understand the crucial candidate genes and related signaling pathways which is associated with the mechanobiology of chondrocytes in OA.

With the developing of modern bioinformatics, an increasing number of evidences showed that many genes and signaling pathways mediate the onset and progression of OA. Ramos et al. [16] demonstrated that 694 Differentially expressed genes (DEGs) mainly enriched in the apoptosis-related pathway in the blood of OA patients seem to be related to the onset of OA. Lambert et al. [17] also identified 896 DEGs between normal and inflamed areas in the knee synovium and suggested the key pathways included cartilage metabolism, inflammation, Wnt signaling and angiogenesis. Therefore, bioinformatics analysis can be a powerful tool to explain the complicated regulatory networks and molecule mechanisms in the pathogenesis of OA.

In this study, we have identified some differentially expressed genes (DEGs) in the cultured chondrocytes from knee and finger joints through reanalyzing the raw microarray data (GEO Series: GSE68038).

Enrichment analysis, proteinprotein interaction (PPI) networks, and module analysis were operated on the DEGs. Afterwards, the overlap between the DEGs in human chondrocytes from knee and finger joints and those in the cartilage from healthy and osteoarthritic joints (GEO Series: GSE117999) was also performed to confirm the role in the osteoarthritis development. Finally, analysis of miRNA regulatory network and

miRNA associated pathway in these overlapped hub genes were also performed to initially provide certain possible therapeutic target for $\mathrm{OA}$.

\section{Methods And Materials}

\section{Microarray data}

GSE68038 gene data is from the GEO database (http://www.ncbi.nlm.nih.gov/geo/), which is expressed on the GPL16686 platform [Affymetrix Human Gene 2.0 ST Array]. In the dataset, three samples of cultured chondrocytes from knee and proximal interphalangeal finger joints, respectively were compared. The chondrocytes were expanded in monolayer at $37^{\circ} \mathrm{C}, 5 \% \mathrm{CO} 2$, and $5 \% \mathrm{O} 2$, in DMEM/F12, $10 \%$ fetal bovine serum and 1\% Penicilin/Streptomycin solution [18]. Additionally, GSE117999 gene data was also obtained. The expression data was generated on GPL20844 platform and Agilent Microarray was used to identify DEGs in cartilage tissues obtained from 12 patients with $\mathrm{OA}$ and from 12 patients without OA.

\section{Identification of differentially expressed genes}


The original microarray data was analyzed by heat mapping using Morpheus (https:// software.broadinstitute.org/morpheus/) which can make gene expression to be observed visually. The data which was divided into the cultured chondrocytes from knee joint group and from proximal interphalangeal joint group were assigned using GEO2R (https://www.ncbi.nlm.nih.gov/geo/geo2r/) to identify the DEGs. DEGs with the cut-off criterion ( $p$ value $<0.05$ and Log2 $|\mathrm{FC}|>1$ ) were taken in to consideration for further analyses. Additionally, the overlapped DEGs were obtained from the GSE68038 and GSE117999 gene data with the cut-off criterion.

\section{Enrichment analysis for DEGs}

Enrichment analyses for DEGs were operated by Metascape (http://metascape.org/gp/index. html) and DAVID 6.8 (https://david.ncifcrf.gov/), respectively. Enrichment analysis include several available terms, such as pathway, functional set, structural complex and signature module in Metascape, and GO enrichment (biological process, cell components, molecular function) and KEGG pathway analysis in DAVID. $P<0.05$ indicating a statistically significant difference was taken into consideration. There were three steps to perform enrichment analyses for DEGs in this study. Firstly, an enrichment analysis was undertaken for DEGs in GSE68038 gene data. Secondly, enrichment analysis for the overlapped DEGs across two data bases was operated. Thirdly, a meta-enrichment analysis for two DEGs lists was conducted.

\section{Protein-protein interaction (PPI) network and module analysis}

A threshold (interaction scores $>0.4$ ) was nessary for the analysis of PPI networks by using the STRING database (http://www.string-db.org), which can provide interactions across with the matched proteins. Visualizing the results from the PPI network was on the base of Cytoscape 3.5 (http://www.cytoscape.org/). Cytoscape plug-in Centiscape was used to judge the centrality of DEGs in PPI networks with three algorithms: degree centrality, betweenness centrality, and closeness centrality. When the centrality degree of DEGs was over 5 , they were defined as key genes in this study. Subsequently, module analysis on the PPI network results was performed by the Cytoscape plug-in MCODE and the module analysis results were used for enrichment analysis.

\section{MiRNA regulatory network and miRNA-associated pathways of the overlapped hub genes}

An online database (miRDB) was used for the perdiction of MiRNAs interacting with ten mRNAs from the overlapped top 10 hub genes (CDK1, KIF23, KIF11 GINS2, BUB1, PRC1, FOXM1, PBK, MCM8 and DBF4). Targets with a score of $>60$ and miRNAs with binding sites less than 1000 were investigated. The construction of miRNA-mRNA regulatory network used Cytoscape. The KEGG analysis for predicted miRNAs from six overlapped hub genes (CDK1, KIF23, KIF11 GINS2, BUB1 and PBK, centrality degree $\geq 10$ ) was also performed on the basis of DIANA-mirPath v3.0 [19], which is a useful network tool that provides experimental support for the interaction of miRNAs-mRNA.

\section{Results}




\section{Identification and enrichment analysis of DEGs in chondrocytes from knee and finger joints}

Morpheus was used to the observation of the overall gene expression of all samples (Fig.1A) was on the use of Morpheus, and differences in gene expressions between the cultured chondrocytes from knee and finger joints were identified. Totally, 213 DEGs were identified by GEO2R analysis, among which 112 were downregulated and 101 were upregulated (Fig.1B).

Subsequently, enrichment analysis (Fig.1C), and GO and KEGG pathway analysis (Fig.2) of the DEGs was also performed. Overall, the majority of the DEGs located in the 'extracellular region', 'extracellular space' and 'proteinaceous extracellular matrix', exert molecular functions by 'heparin binding', 'kinase activity' and 'growth factor activity', and regulate biological process by 'positive or negative regulation of cell proliferation', 'embryonic limb morphogenesis' and 'positive regulation of cell division'. Furthermore, the DEGs mainly involve in these pathways of "P13K-Akt signaling pathway", "Rap1 signaling pathway" and "Ooctyte meiosis".

\section{PPI network analysis of DEGs in chondrocytes from knee and finger joints}

Following the analysis which is on the basis of the PPI networks, 62 nodes and 339 edges were identified in Cytoscape (Fig.3A). Centiscape was used to analyze the PPI network and the gene with centrality degree $\geq 5$ were defined as hub gene. The top 10 hub genes were CCNB1, SHCBP1, BUB1, CKD1, KIF20A, PBK, CKAP2L, IGF-1, TTK, MKI67.

Also, six modules totally were selected by using MCODE analysis (Fig.3A), two of which (module 1 and 2) consist of hub genes. Enrichment analysis suggested that module 1 and 2 is likely to be associated with 'chromosome segregation', 'mitotic cell cycle,' and 'apoptotic signaling pathway'. The detailed results of module 1 were shown in Table 1, and the detailed results of module 2 were shown in Table 2.

\section{Genetic overlap with DEGs in cartilage from healthy and osteoarthritic joints}

To confirm the role of the genes in the OA development, the overlap between the DEGs in the human chondrocytes from knee and finger joints and those in the cartilage from healthy and osteoarthritic joints. In total, the two GEO datasets shared 122 intersection genes, among which 49 were upregulated and 73 were downregulated jointly (Fig.4A).

The overlapped DEGs mainly enriched in DNA replication and mitotic nuclear division (Biological process), microtubule binding and calcium ion binding (Cellular component), nucleoplasm and mototic spindle (molecular function) (Fig.4B and 4C).

\section{Analysis of hub genes and modules in the overlapped genes}

Following the analysis which is on the basis of the PPI networks, 20 nodes and 213 edges were identified in (Fig. 4). The genes with centrality degree $\geq 5$ were defined as hub genes, included CDK1, 
KIF11, KIF23, BUB1, MAD2L1, KIAA0101, PBK, GINS2, PRC1, FOXM1, DBF4 and MCM8 (Figure 5B).

The hub genes were downregulated jointly in the two datasets (Fig. 5D), and mainly enriched in mitotic cell cycle, regulation of cell cycle process, DNA replication, cell cycle and microtubule cytoskeleton organization (Fig. 5E).

\section{Analysis of miRNA regulatory network and miRNA associated pathways in the overlapped genes}

The miRNA-regulatory network in the top 10 overlapped hub genes (CDK1, KIF23, KIF11 GINS2, BUB1, PRC1, FOXM1, PBK, MCM8 and DBF4) was also identified in this study. 120 predicted miRNAs had been idenfitified, including miR-146a-5p, miR-19b-3p, miR-122-5p and MiR-15a-5p (Fig. 6A). In addition, enrichment analysis of these predicted miRNAs from top 6 overlapped hub genes (CDK1, KIF23, KIF11 GINS2, BUB1 and PBK, centrality degree $\geq 10$ ) was performed and found that the OA-related pathways included TGF- $\beta$ signaling pathway, Wnt signaling pathway, MAPK signaling pathway, Rap1 signaling pathway and P13K-Akt signaling pathway (Fig. 6B).

\section{Discussion}

Knee OA is a common degenerative joint disease, which pathologically characterized by the progressive degeneration of articular cartilage. Mechanical stress may lead to alterations in the composition, structure, and metabolism of articular cartilage, therefore it was well known to be a significant risk factor for the onset and progression of OA [20]. In the current study, to explore the action mechanism of mechanical stress on the osteoarthritic development, a three-step approach was followed to perform bioinformatics analysis. Firstly, in total 213 DEGs were identified in cultured chondrocytes from knee and finger joints, of which 101 genes were upregulated and 112 were downregulated and then enrichment analysis was performed. Subsequently, 122 overlapped genes between the DEGs in the chondrocytes from knee and finger joints and those in the cartilage from healthy and osteoarthritic joints were identified, and then an enrichment analysis was performed for further understanding the regulating roles of the intersection genes in OA. Finally, analysis of miRNA regulatory network and miRNA associated pathway in the overlapped hub genes were also performed.

Compared to the proximal interphalangeal joint, the knee joint, a foremost weight-bearing joint, is continuously subjected to mechanical loading. During standing statically, the mechanical stress applied to human knee joints is about 3.5 MPa [21], while the compressive stress during activities such as stair climbing will rise up to $20 \mathrm{MPa}$ [22]. The mechanical loading generated during daily activity is a fundamental stimulus for the activity of chondrocytes, physiologically necessary for maintaining cell homeostasis, while pathologically caused significant damage to the articular cartilage [23]. In this study, enrichment analysis of DEGs in cultured chondrocytes from knee and finger joints also revealed the dual character of biological process: positive or negative regulation of cell proliferation, positive regulation of cell division, positive regulation of apoptotic process and embryonic limb morphogenesis. A number of key transduction mechanisms have been identified that facilitate the mechanically-driven enhancement of chondrocyte homeostasis, including ion channel [24,25], integrins signaling [26] and primary cilia [27]. 
Correspondingly, the current study also revealed that DEGs exert molecular function through regulating heparin binding, growth factor activity, calcium ion binding and integrin binding. Meanwhile, the KEGG pathways analyzed by DEGs included 'PI3K-Akt signaling pathway', 'FoxO signaling pathway', 'Ras signaling pathway', 'Cell cycle' and 'Oocyte meiosis', which showed the consistence with the findings of previous studies $[28,29,30,31]$.

PPI networks with the DEGs were constructed as well, and two modules included hub genes were also identified. Module 1 included Kinesin-like protein (KIF18A), KIF15, DNA topoisomerase (TOP2A), budding uninhibited by benzimidazoles (BUB1) and Cyclin-dependent kinase (CDK1), mainly involved in such biological processes as chromosome segregation, cell cycle and mitotic cell division, which agree with the results shown in the enrichment analysis. Module 2 including hub gene insulin-like growth factor 1 (IGF1) and vascular endothelial growth factor (VEGF) was associated with "platelet degranulation", "protein processing", "regulation of protein secretion" and "apoptotic signaling pathway". These results are consistent with the previous studies indicating that mechanical loading directly induce weight-bearing area hypoxia followed by upregulated expression of VEGF [32], Shear stress induced the activation of IGF1 pathway which involved in the regulation of morphology and proliferation in the chondrocytes [33], and even IGF-1 has been shown to protect chondrocytes from apoptosis [34]. Collectively, mechanical stress has multiple influences on joint and cartilage, such as good ones including promoting cartilage development and maintaining chondrocyte homeostasis, or bad ones including inhibiting chondrocyte proliferation and accelerating cell apoptosis.

To confirm the effect of mechanical stress in the osteoarthritis development, the overlap between the DEGs in the chondrocytes from knee and finger joints and those in the cartilage from healthy and osteoarthritic joints was also performed. In total, we screened out 122 jointly expressive intersection genes, brought a main enrichment in DNA replication, cell division, negative regulation of stress-activated MAPK cascade, calcium ion binding and positive regulation of apoptotic process. In general, the loss of cartilage homeostasis and the dysfunction of chondrocytes phenotypes which includes cell apoptosis, cell division and proliferation, are the important pathological process of OA [35,36]. This study also suggested that the overlapped DEGs may involve in such biological process as DNA replication, cell division and positive regulation of apoptotic process. Meanwhile, the MAPK signaling pathway has been found to receive, transduce and convey mechanical information to the chondrocyte interior [37], which also evidenced by the enrichment of negative regulation of stress-activated MAPK cascade in the overlapped genes. Intracellular calcium signaling is among the earliest responses of chondrocytes to physical stimuli and plays an important role in the mechanobiology of chondrocytes in a natural residing environment [38]. Here, calcium ion binding was also found to be the significant enrichment for the DEGs, which suggested the role of calcium ion channel and downstream pathway in the mechanical stressinduced osteoarthritic development.

Additionally, many overlapped hub genes were identified in the PPI analysis, and submodule including hub genes CDK1 and Forkhead box M1 (FOXM1) was also selected, which enriched in cell cycle, regulation of cell cycle process and DNA replication. Cell cycle regulation is critical for chondrocyte 
differentiation and hypertrophy. Due to terminal differentiation is usually accompanied with cell cycle exit and the non-proliferative maintenance, cell cycle arrest is required for chondrocyte transition from proliferation to hypertrophic differentiation [39,40], which played a vital role in early and late stage OA $[41,42]$. In the present study, significantly, the hub genes involved in cell cycle were entirely downregulated, which suggested the abnormal regulation of cell cycle process, implicating for chondrocyte differentiation and hypertrophy. CDK1, a member of the Ser/Thr protein kinase family, functions as a molecular switch from proliferation to hypertrophic differentiation of chondrocytes. Therefore, CDK1 is usually highly expressed in columnar proliferative chondrocytes and greatly downregulated upon differentiation into hypertrophic chondrocytes [43]. FOXM1 is a transcription factor that acts as a regulator of the cell cycle [44], which may involve in such biological process as mitotic cell cycle, Wnt signaling and apoptosis [45]. In addition, the role of overlapped hub genes including KIF11, BUB1 and MAD2L1 in the pathogenesis of OA was also found in the previous studies $[46,47]$. Accordingly, under mechanical stress, the cell cycle-related genes were downregulated, as a result, chondrocyte cell proliferation would be blocked and transformed to differentiation and hypertrophy, which may be important in the pathogenesis of OA.

Finally, the miRNA-regulatory network in the overlapped hub genes was also identified in this study. 120 predicted miRNAs were identified, including miR-146a-5p, miR-19b-3p, miR-122-5p and MiR-15a-5p, which were mainly enriched in TGF- $\beta$ signaling pathway, Wnt signaling pathway and MAPK signaling pathway. Actually, these predicted miRNAs and pathways played important roles in OA has been verified in previous studies. Zhang et al have suggested that mechanical stress contributes to osteoarthritis development through the activation of TGF- $\beta$ signaling pathway [5]. In addition, some professores suggested that circulating miRNAs, for example, miR-19b-3p and miR-122-5p, are promising biomarkers to diagnose knee OA [48, 49]. Furthermore, Chen et.al also suggested that MiR-15a-5p regulated the viability of human osteoarthritis chondrocytes via targeting VEGF [50]. Thus, the critical miRNAs and signaling pathways can be used as new targets for OA diagnosis and therapy, and can make some contributions for further experimental studies on the part of potential hallmarks.

In conclusion, mechanical stress has shown the multiple influences on chondrocytes and cartilage, physiologically necessary for maintaining chondrocyte homeostasis and promoting cartilage development. While, pathologically, mechanical stress could induce cartilage destruction and advance osteoarthritic development via boosting chondrocyte differentiation and hypertrophy, and accelerating cell apoptosis. Additionally, TGF- $\beta$ signaling pathway, Wnt signaling pathway and MAPK signaling pathway is likely related to mechanical stress-induced OA progression and can be the potential biomarkers and therapeutic targets, which needs further experiment to verify and provide useful evidence for future researches in OA.

\section{Declarations}

\section{Ethics approval and consent to participate}


Not applicable

\section{Consent for publication}

This is an open-access article distributed under the terms of the Creative Commons Attributions licence (CC-BY-NC), which permits unrestricted use, distribution, and reproduction in any medium, but not for commercial gain, provided the original author and source are credited

\section{Availability of data and material}

The microarray data GSE68038 and GSE117999 were downloaded from the GEO database in NCBI (http://www.ncbi.nlm.nih.gov/geo/).

\section{Funding}

This research was supported by the National Natural Science Foundation of China (81774334, 81873329), Postgraduate Research \& Practice Innovation Program of Jiangsu Province (KYCX18_1551), The China Scholarship Council (NO.201808320331).

\section{Competing interests}

The authors declare that there is no conflict of interests regarding the publication of this paper.

\section{Authors' contributions}

W Mei and SJ Yin: design study and perform the statistical analysis; RL Xing, L Zhang and XQ Shi: analyze the data and prepare the figure; NS Zhang, XC Li, PM Wang, GC Huang: design the study and edit the manuscript.

\section{Acknowledgements}

Not applicable.

\section{References}

1. Musumeci G, Szychlinska MA and Mobasheri A: Age-related degeneration of articular cartilage in the pathogenesis of osteoarthritis: molecular markers of senescent chondrocytes. Histol Histopathol 30:1-12, 2015.

2. Liu Q, Hu X, Zhang X, Duan X, Yang P, Zhao F and Ao Y: Effects of mechanical stress on chondrocyte phenotype and chondrocyte extracellular matrix expression. Sci Rep 17: 37268, 2016.

3. Ramage L, Nuki G and Salter DM: Signalling cascades in mechanotransduction: Cell-matrix interactions and mechanical loading. Scand J Med Sci Sports 19: 457-469, 2009.

4. Yang $Y$, Wang $Y$, Kong $Y$, Zhang $X$, Zhang $H$, Gang $Y$ and Bai L: Mechanical stress protects against osteoarthritis via regulation of the AMPK/NF-KB signaling pathway. J Cell Physiol 12, 2018. 
5. Zhang RK, Li GW, Zeng C, Lin CX, Huang LS, Huang GX, Zhao C, Feng SY and Fang H: Mechanical stress contributes to osteoarthritis development through the activation of transforming growth factor beta 1 (TGF- $\beta 1$ ). Bone Joint Res 7: 587-594, 2018.

6. Sun $L$, Zhao J, Wang $H$, Pan $Y$, Wang $L$ and Zhang WB: Mechanical stress promotes matrix synthesis of mandibular condylar cartilage via the RKIP-ERK pathway. J Mol Histol 48: 437-446, 2017.

7. Huang Z, Zhou M, Wang Q, Zhu M, Chen S and Li H: Mechanical and hypoxia stress can cause chondrocytes apoptosis through over-activation of endoplasmic reticulum stress. Arch Oral Biol 84: 125-132, 2017.

8. Chen D, Shen J, Zhao W, Wang T, Han L, Hamilton JL and Im HJ: Osteoarthritis: toward a comprehensive understanding of pathological mechanism. Bone Res 17: 16044, 2017.

9. Wallace IJ, Worthington S, Felson DT, Jurmain RD, Wren KT, Maijanen H, Woods RJ and Lieberman DE: Knee osteoarthritis has doubled in prevalence since the mid-20th century. Proc Natl Acad Sci USA114: 9332-9336, 2017.

10. Vos T, et al: Years lived with disability (YLDs) for 1160 sequelae of 289 diseases and injuries 19902010: A systematic analysis for the Global Burden of Disease Study 2010. Lancet 380: 2163-2196, 2012.

11. Felson DT: Osteoarthritis as a disease of mechanics. Osteoarthritis Cartilage 21:10-15, 2013.

12. Asay JL, Erhart-Hledik JC and Andriacchi TP: Changes in the total knee joint moment in patients with medial compartment knee osteoarthritis over 5 years. J Orthop Res 36: 2373- 2379, 2018.

13. Sanchez-Adams J, Leddy HA, McNulty AL, O'Conor CJ and Guilak F. The mechanobiology of articular cartilage: bearing the burden of osteoarthritis. Curr Rheumatol Rep 16: 451, 2014.

14. Bomer N, Cornelis FM, Ramos YF, den Hollander W, Storms L, van der Breggen R, Lakenberg N, Slagboom PE, Meulenbelt I and Lories RJ: The effect of forced exercise on knee joints in Dio2(-/-) mice: type II iodothyronine deiodinase-deficient mice are less prone to develop OA-like cartilage damage upon excessive mechanical stress. Ann Rheum Dis 75: 571-577, 2016.

15. Visser AW, de Mutsert R, le Cessie S, den Heijer M, Rosendaal FR, Kloppenburg M and NEO Study Group: The relative contribution of mechanical stress and systemic processes in different types of osteoarthritis: the NEO study. Ann Rheum Dis 74: 1842-1847, 2015.

16. Ramos YF, Bos SD, Lakenberg N, Bohringer S, den Hollander WJ, Kloppenburg M, Slagboom PE and Meulenbelt I: Genes expressed in blood link osteoarthritis with apoptotic pathways. Ann Rheum Dis 73: 1844-1853, 2014.

17. Lambert C, Dubuc JE, Montell E, Vergés J, Munaut C, Noël A and Henrotin Y: Gene expression pattern of cells from inflamed and normal areas of osteoarthritis synovial membrane. Arthritis Rheumatol 66: 960-968, 2014.

18. Stradner MH, Dreu M, Angerer H, Gruber G, Wagner K, Peischler D, Krischan V, Leithner A, Anderhuber F, Graninger WB. Chondrocyte cultures from human proximal interphalangeal finger joints. J Orthop Res 34: 1569-1575, 2016. 
19. Vlachos IS, Zagganas K, Paraskevopoulou MD, Georgakilas G, Karagkouni D, Vergoulis T, Dalamagas T and Hatzigeorgiou AG: Diana-mirpath v3.0: Deciphering microrna function with experimental support. Nucleic Acids Res 43: W460-466, 2015.

20. Griffin TM and Guilak F. The role of mechanical loading in the onset and progression of osteoarthritis. Exerc Sport Sci Rev 33:195-200, 2005.

21. Herberhold C, Faber S, Stammberger T, Steinlechner M, Putz R, Englmeier KH, Reiser M, and Eckstein $\mathrm{F}$ : In situ measurement of articular cartilage deformation in intact femoropatellar joints under static loading. J Biomech 32: 1287-1295, 1999.

22. Grodzinsky AJ, Levenston ME, Jin M and Frank EH: Cartilage tissue remodeling in response to mechanical forces. Annu Rev Biomed Eng 2: 691-713, 2000.

23. Cattano NM, Driban JB, Cameron KL and Sitler MR: Impact of physical activity and mechanical loading on biomarkers typically used in osteoarthritis assessment: current concepts and knowledge gaps. Ther Adv Musculoskelet Dis 9:11-21, 2017.

24. 2 Phan MN1, Leddy HA, Votta BJ, Kumar S, Levy DS, Lipshutz DB, Lee SH, Liedtke W and Guilak F: Functional Characterization of TRPV4 as an Osmotically Sensitive Ion Channel in Porcine Articular Chondrocytes. Arthritis Rheum 60: 3028-3037, 2009.

25. Servin-Vences MR, Richardson J, Lewin GR and Poole K: Mechanoelectrical transduction in chondrocytes. Clin Exp Pharmacol Physiol 45: 481-488, 2018.

26. Millward-Sadler SJ and Salter DM: Integrin-dependent signal cascades in chondrocyte mechanotransduction. Ann Biomed Eng 32: 435-446, 2004.

27. Wann AK, Zuo N, Haycraft CJ, Jensen CG, Poole CA, McGlashan SR and Knight MM: Primary cilia mediate mechanotransduction through control of ATP-induced $\mathrm{Ca}^{2+}$ signaling in compressed chondrocytes. Faseb Journal: 1663-1671, 2012.

28. He P, Shen N, Gao G, Jiang X, Sun H, Zhou D, Xu N, Nong L and Ren K: Periodic Mechanical Stress Activates PKC $\delta$-Dependent EGFR Mitogenic Signals in Rat Chondrocytes via PI3K-Akt and ERK1/2. Cell Physiol Biochem 39: 1281-1294, 2016.

29. Ma Y and Wang H: PI3K/Akt/FoxO: a novel participant in signal transduction in bone cells under mechanical stimulation. Cell Biol Int 36: 923-926, 2012.

30. JJin G, Sah RL, Li YS, Lotz M, Shyy JY and Chien S: Biomechanical regulation of matrix metalloproteinase-9 in cultured chondrocytes. Orthop Res 18: 899-908, 2000.

31. Zhu F, Wang P, Lee NH, Goldring MB and Konstantopoulos K: Prolonged application of high fluid shear to chondrocytes recapitulates gene expression profiles associated with osteoarthritis. PLoS One 5: e15174, 2010.

32. Yu J, Liang F, Huang H, Pirttiniemi P and Yu D: Effects of loading on chondrocyte hypoxia, HIF-1a and VEGF in the mandibular condylar cartilage of young rats. Orthod Craniofac Res 21: 41-47, 2018.

33. Tahimic CG, Long RK, Kubota T, Sun MY, Elalieh H, Fong C, Menendez AT, Vilardaga JP and Bikle DD: Regulation of Ligand and Shear Stress-induced Insulin-like Growth Factor 1 (IGF1) Signaling by the 
Integrin Pathway. J Biol Chem 291: 8140-8149, 2016.

34. Higgins TF and Johnson BD: Effect of exogenous IGF-1 on chondrocyte apoptosis in a rabbit intraarticular osteotomy model. J Orthop Res 28: 125-130, 2010.

35. Hu W, Zhang W, Li F, Guo F and Chen A. Mir-139 is up-regulated in osteoarthritis and inhibits chondrocyte proliferation and migration possibly via suppressing eif4g2 and igf1r. Biochem Biophys Res Commun 474: 296-302, 2016.

36. O'Connell GD, Tan AR, Cui V, Bulinski JC, Cook JL, Attur M, Abramson SB, Ateshian GA and Hung CT: Human chondrocyte migration behaviour to guide the development of engineered cartilage. $\mathrm{J}$ Tissue Eng Regen Med 11: 877-886, 2017.

37. Fanning PJ, Emkey G, Smith RJ, Grodzinsky AJ, Szasz N and Trippel SB: Mechanical regulation of mitogen-activated protein kinase signaling in articular cartilage. J Biol Chem 19: 50940-50948, 2003.

38. Lv M, Zhou Y, Chen X, Han L, Wang L and Lu XL: Calcium signaling of in situ chondrocyte in articular cartilage under compressive loading: Roles of calcium sources and cell membrane ion channels. $J$ Orthop Res 36: 730-738, 2018.

39. Negishi Y, Ui N, Nakajima M, Kawashima K, Maruyama K, Takizawa T and Endo H: p21Cip-1/SDI1/WAF-1 gene is involved in chondrogenic differentiation of ATDC5 cells in vitro. J Biol Chem 276, 33249-33256, 2001.

40. LuValle P and Beier F: Cell cycle control in growth plate chondrocytes. Front Biosci 5, D493-503, 2000.

41. Huebner JL, Johnson KA, Kraus VB and Terkeltaub RA: Transglutaminase 2 is a marker of chondrocyte hypertrophy and osteoarthritis severity in the Hartley guinea pig model of knee OA. Osteoarthritis Cartilage 17:1056-1064, 2009.

42. van der Kraan PM and van den Berg WB: Chondrocyte hypertrophy and osteoarthritis: role in initiation and progression of cartilage degeneration? Osteoarthritis Cartilage 2012: 223-232, 2012.

43. Saito M, Mulati M, Talib SZ, Kaldis P, Takeda S, Okawa A and Inose H: The Indispensable Role of Cyclin-Dependent Kinase 1 in Skeletal Development. Sci Rep 6: 20622, 2016.

44. Wang IC, Chen YJ, Hughes DE, Ackerson T, Major ML, Kalinichenko VV, Costa RH, Raychaudhuri P, Tyner AL and Lau LF: FoxM1 regulates transcription of JNK to promote the G1/S transition and tumor cell invasiveness. J Biol Chem 283:20770-20778, 2008.

45. Dunn SL, Soul J, Anand S, Schwartz JM, Boot-Handford RP and Hardingham TE: Gene expression changes in damaged osteoarthritic cartilage identify a signature of non- chondrogenic and mechanical responses. Osteoarthritis Cartilage 24: 1431-1440, 2016.

46. Sun J, Yan B, Yin W and Zhang X: Identification of genes associated with osteoarthritis by microarray analysis. Mol Med Rep 12: 5211-5216, 2015.

47. Yang $J$ and Wang N: Genome-wide expression and methylation profiles reveal candidate genes and biological processes underlying synovial inflammatory tissue of patients with osteoarthritis. Int $\mathrm{J}$ Rheum Dis 18: 783-790, 2015. 
48. Ntoumou E, Tzetis M, Braoudaki M, Lambrou G, Poulou M, Malizos K, Anastasopoulou L and Tsezou A: Serum microrna array analysis identifies mir-140-3p, mir-33b-3p and mir-671-3p as potential osteoarthritis biomarkers involved in metabolic processes. J Cell Biochem 9: 127, 2017.

49. Kong R, Gao J, Si Y and Zhao D: Combination of circulating mir-19b-3p, mir-122-5p and mir-486-5p expressions correlates with risk and disease severity of knee osteoarthritis. Am J Transl Res 9: 28522864, 2017.

50. Chen $\mathrm{H}$ and Tian $\mathrm{Y}$ : MiR-15a-5p regulates viability and matrix degradation of human osteoarthritis chondrocytes via targeting VEGFA. Biosci Trends 10: 482-488, 2017.

\section{Tables}

Table 1. Enrichment analysis of module 1 in hub DEGs in chondrocytes from knee and finger joint

\begin{tabular}{|c|c|c|c|c|c|}
\hline Category & Description & Count & $\%$ & $\log 10(P)$ & $\log 10(q)$ \\
\hline GO Biological & chromosome segregation & 12 & 57.14 & -17.28 & -12.97 \\
\hline \multicolumn{6}{|l|}{ Processes } \\
\hline & Cell Cycle, Mitotic & 11 & 52.38 & -12.77 & -9.06 \\
\hline \multicolumn{6}{|l|}{ Reactome Gene Sets } \\
\hline & cell division & 11 & 52.38 & -12.34 & -8.78 \\
\hline \multicolumn{6}{|l|}{ GO Biological } \\
\hline Processes & microtubule-based process & 11 & 52.38 & -11.26 & -8.09 \\
\hline GO Biological & PID PLK1 PATHWAY & 5 & 23.81 & -9.4 & -6.38 \\
\hline Processes & regulation of G2/M transition of cell cycle meiotic cell & 5 & 23.81 & -6.75 & -4.19 \\
\hline Canonical Pathways & cycle & 5 & 23.81 & -5.7 & -3.3 \\
\hline GO Biological & Kinesins & 3 & 14.29 & -4.79 & -2.46 \\
\hline Processes & mitotic cytokinesis & & & & \\
\hline & & 3 & 14.29 & -4.27 & -2 \\
\hline \multicolumn{6}{|l|}{ GO Biological } \\
\hline \multicolumn{6}{|l|}{ Processes } \\
\hline \multicolumn{6}{|l|}{ Reactome Gene Sets } \\
\hline \multicolumn{6}{|l|}{ GO Biological } \\
\hline Processes & & & & & \\
\hline
\end{tabular}

Note. Top 9 clusters with their representative enriched terms (one per cluster). "Count" is the number of genes in the lists with membership in the given ontology term. "\%" is the percentage of all of the genes that are found in the given ontology term (only input genes with at least one ontology term annotation are included in the calculation). "Log10(P)" is the p-value in log base 10. "Log10(q)" is the multi-test adjusted p-value in log base 10. 
Table 2. Enrichment analysis of module 2 in hub DEGs in chondrocytes from knee and finger joint

\begin{tabular}{lccccc}
\hline \multicolumn{1}{c}{ Category } & Description & Count & $\%$ & Log10(P) & Log10(q) \\
\hline GO Biological Processes & platelet degranulation & 5 & 100 & -11.41 & -7.47 \\
GO Biological Processes & protein processing & 3 & 60 & -4.68 & -1.21 \\
GO Biological Processes & regulation of protein secretion & 3 & 60 & -4.23 & -0.87 \\
GO Biological Processes & apoptotic signaling pathway & 3 & 60 & -3.86 & -0.66 \\
Canonical Pathways & NABA MATRISOME ASSOCIATED & 3 & 60 & -3.54 & -0.48 \\
\hline
\end{tabular}

Note. Top 5 clusters with their representative enriched terms (one per cluster). "Count" is the number of genes in the lists with membership in the given ontology term. "\%" is the percentage of all of the genes that are found in the given ontology term (only input genes with at least one ontology term annotation are included in the calculation). "Log10(P)" is the p-value in log base 10 . "Log10(q)" is the multi-test adjusted p-value in log base 10.

\section{Figures}



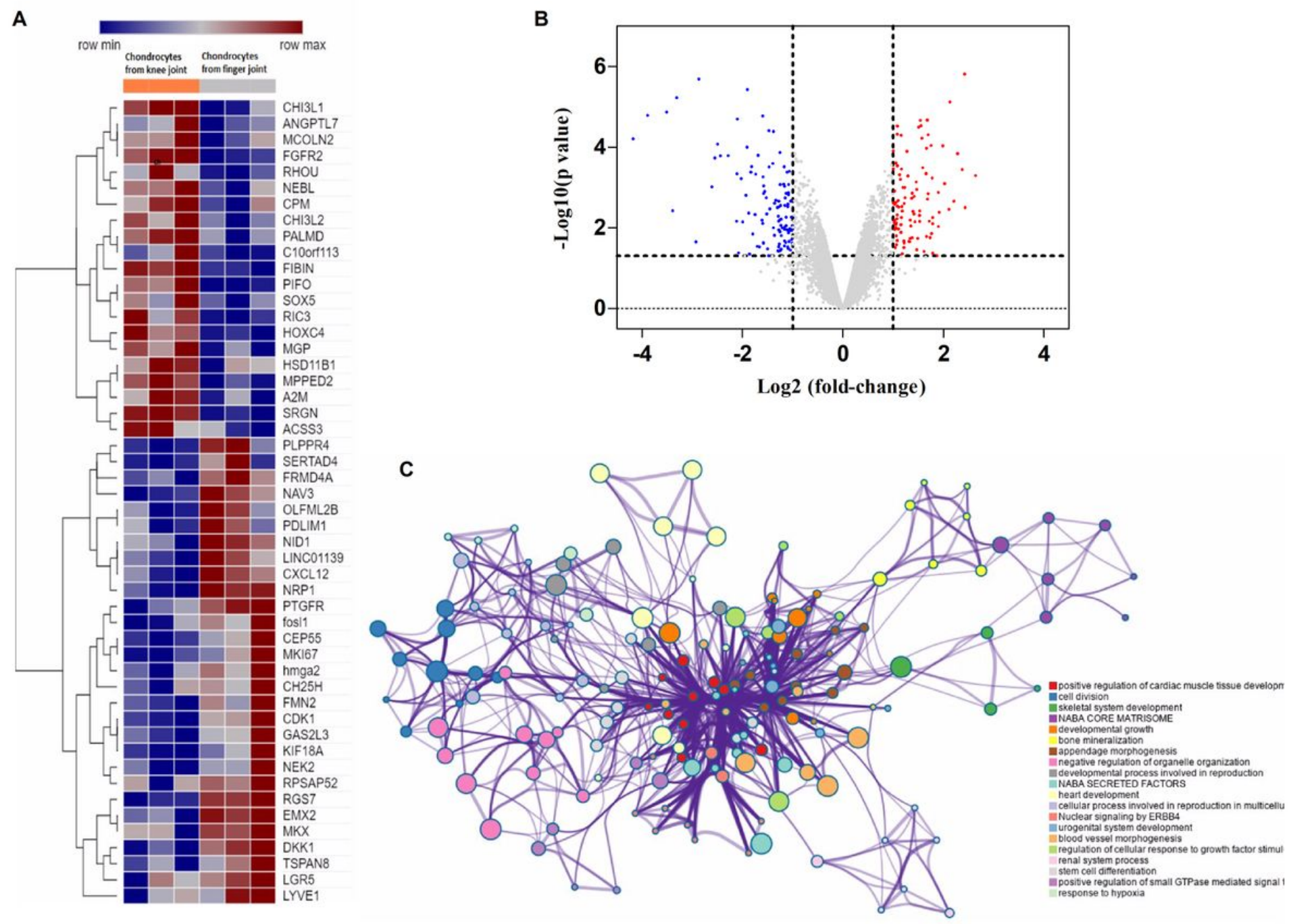

Figure 1

Identification and enrichment analysis of DEGs in chondrocytes from knee and finger joints. (A) Heat map of 50 genes from all samples. Red represents higher gene expression and blue represents lower gene expression. (B) Volcanic map of all genes. Red dots represent upregulated genes, blue dots represent downregulated genes and gray dots represent unregulated genes. (C) Enrichment analysis of DEGs in human chondrocytes between from knee and finger joints, which was produced by Metascape. 

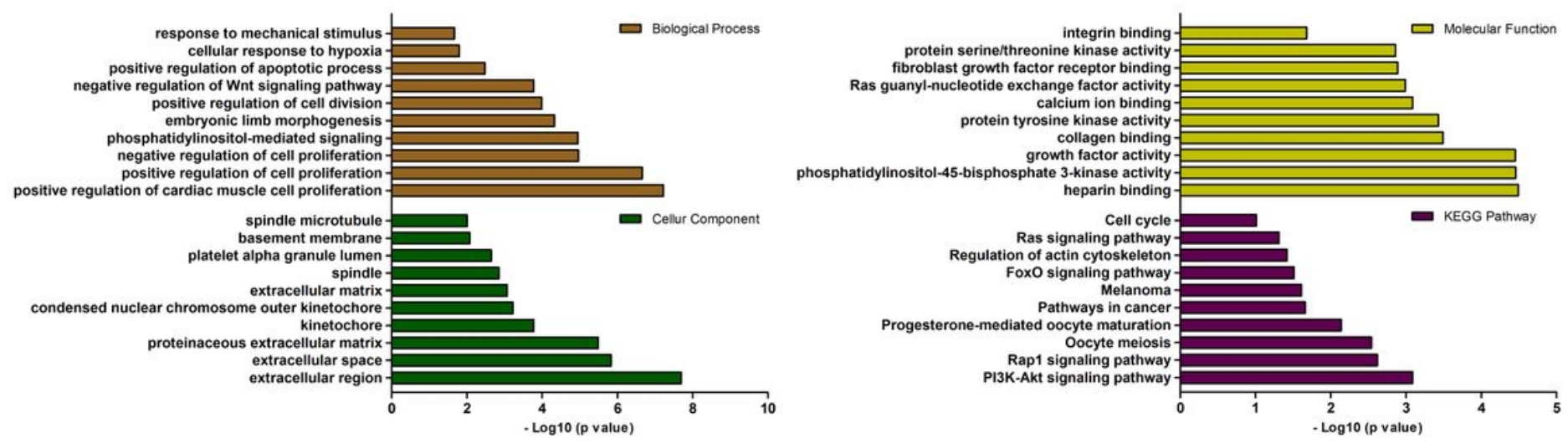

Figure 2

GO enrichment and KEGG pathway analysis of DEGs in chondrocytes from knee and finger joints. The top 10 biological processes, cellular component, molecular function and KEGG pathway analysis of DEGs in human chondrocytes between from knee and finger joints, which was produced by DAVID.

A

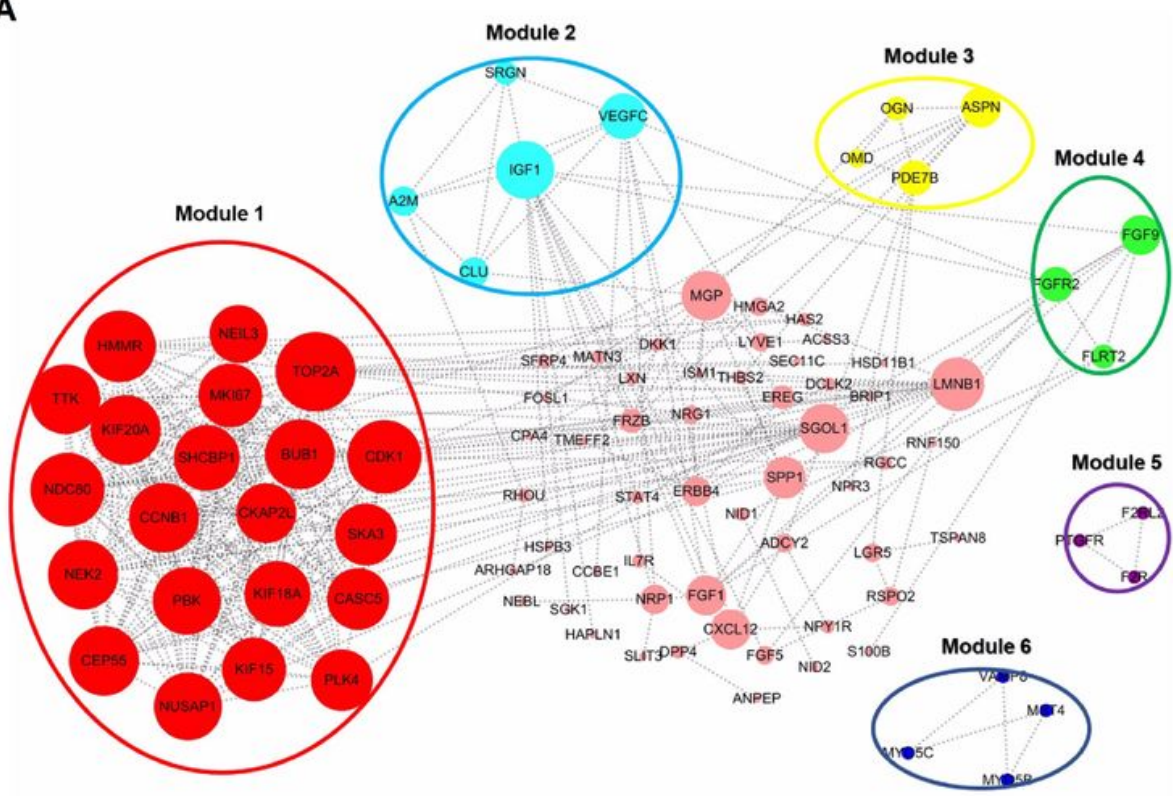

B
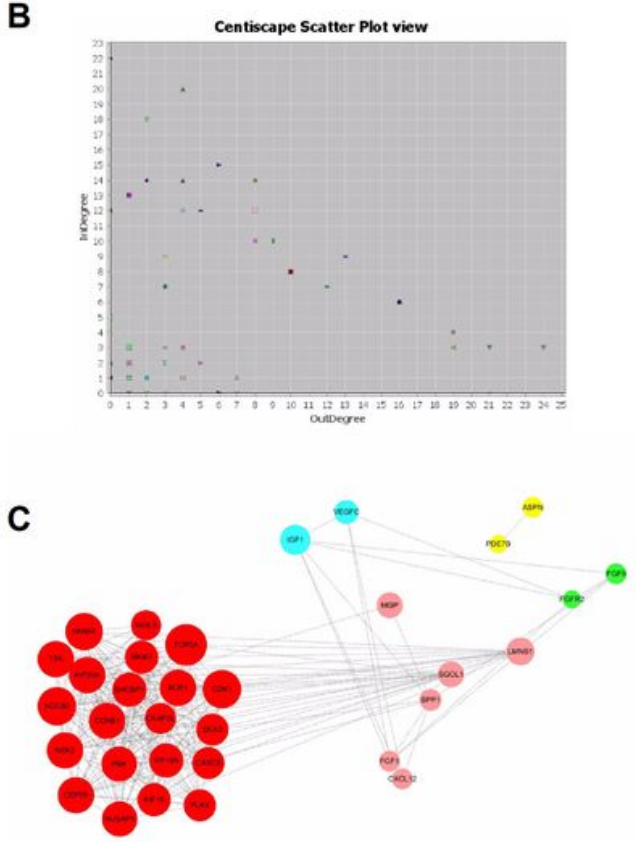

\section{Figure 3}

PPI network analysis of DEGs in chondrocytes from knee and finger joints. (A) PPI network of DEGs constructed using Cytoscape. Sizes of dots are proportional to the score and gray edges indicate protein interactions. (B) Scatter plot view of centrality degree. X-axis represents centrality outdegree and y-axis represents centrality indegree. (C) PPI network of hub genes (centrality degree $\geq 5$ ) constructed using Cytoscape. 
A

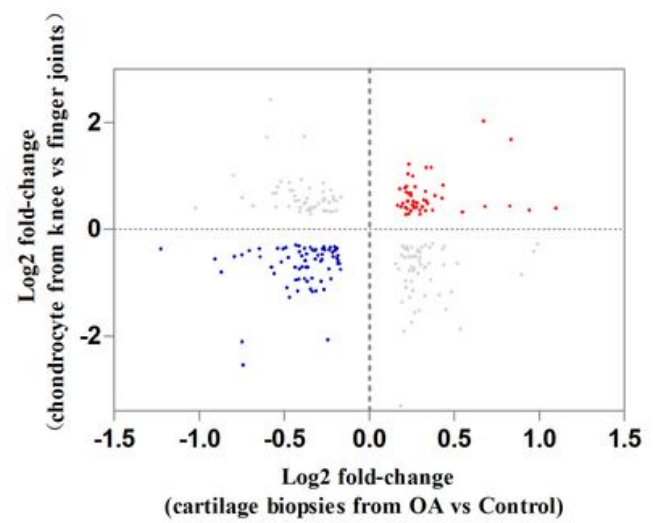

\section{C}

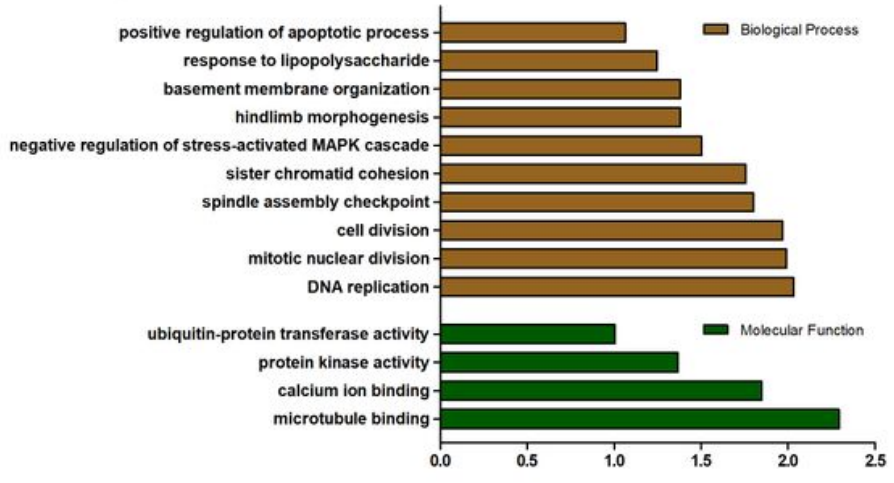

B
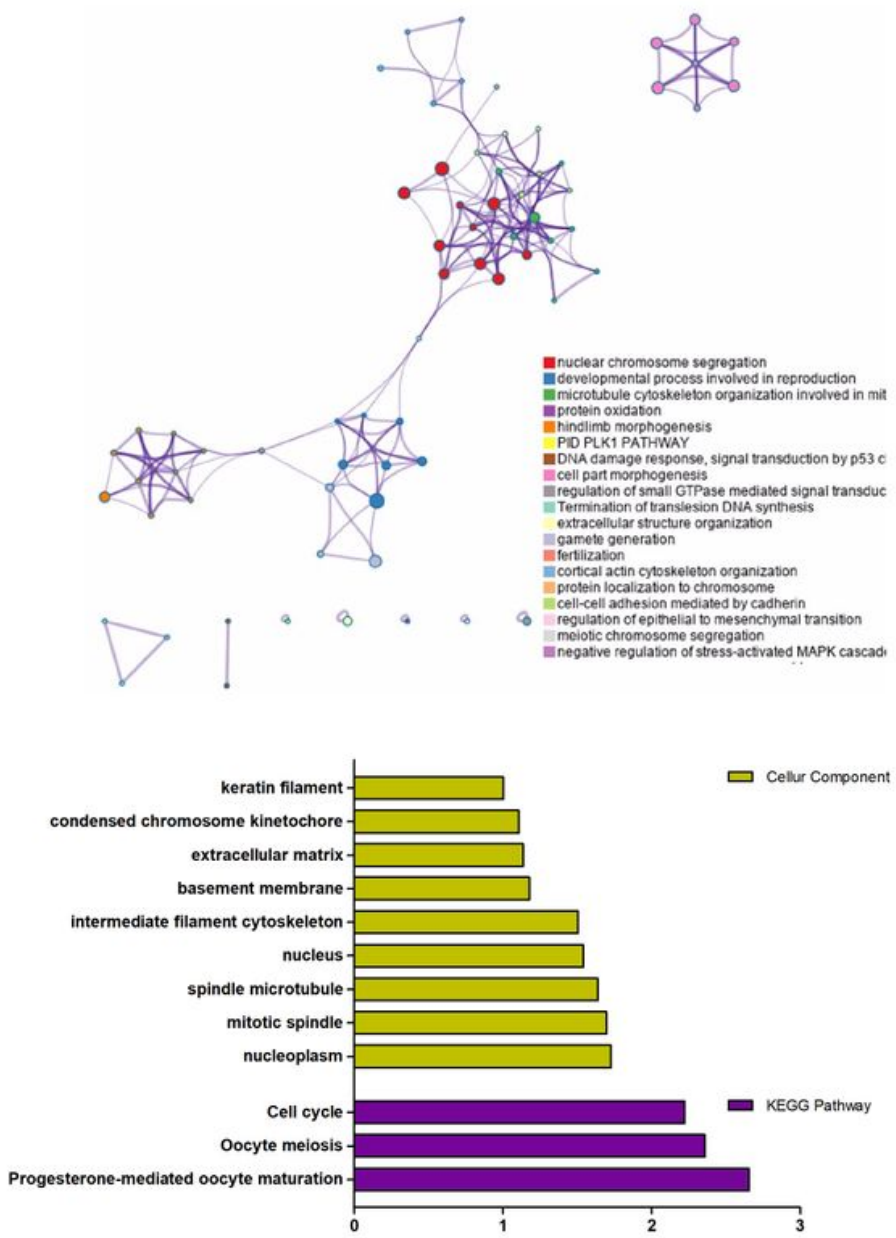

\section{Figure 4}

Genetic overlap between the DEGs in the human chondrocytes from knee and finger joints and those in cartilage from healthy and osteoarthritic joints. (A) Scatter plot view of overlapped genes. Red dots indicate upregulated genes, blue dots indicate downregulated genes. (B) Enrichment analysis of DEGs in human chondrocytes between from knee and finger joints, which was produced by Metascape. (C) GO enrichment and KEGG pathway analysis of DEGs in human chondrocytes between from knee and finger joints, which was produced by DAVID. 
A

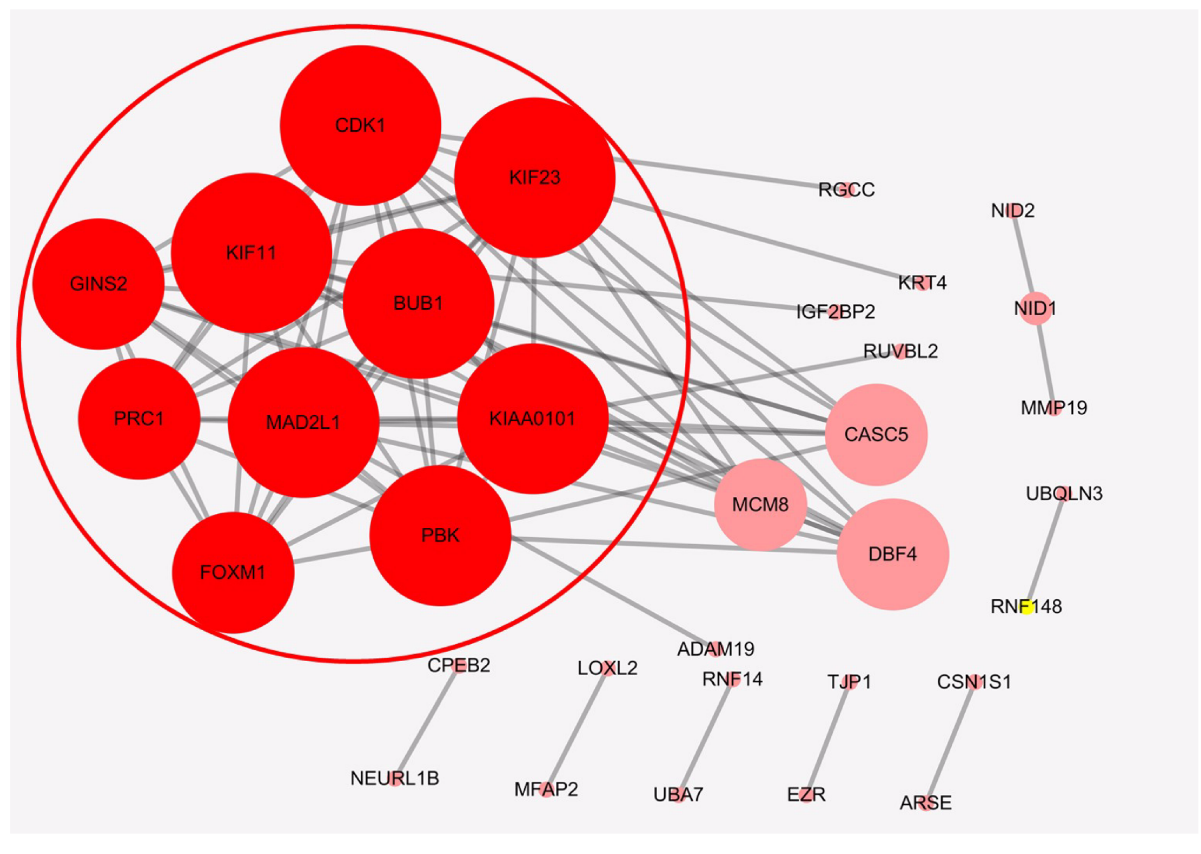

B

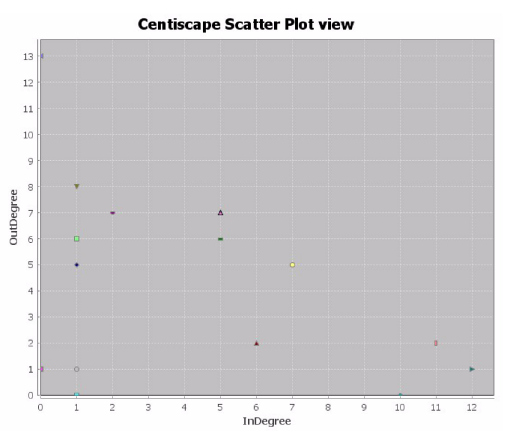

C

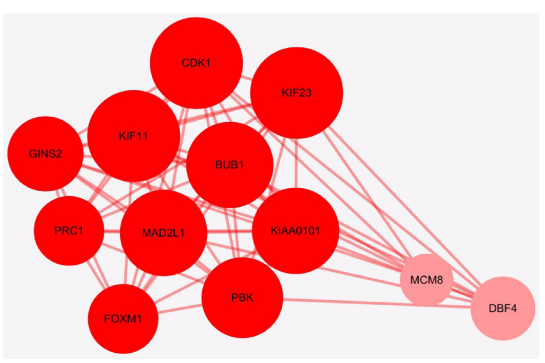

D

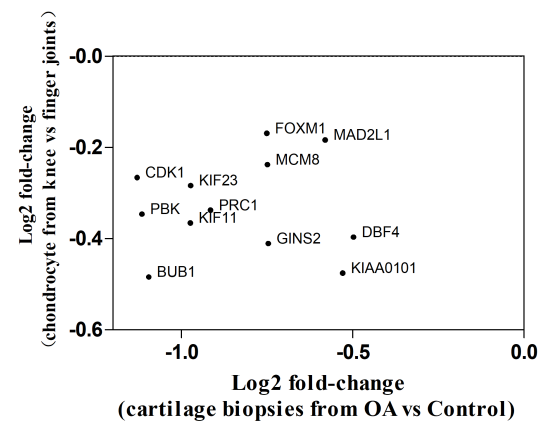

E

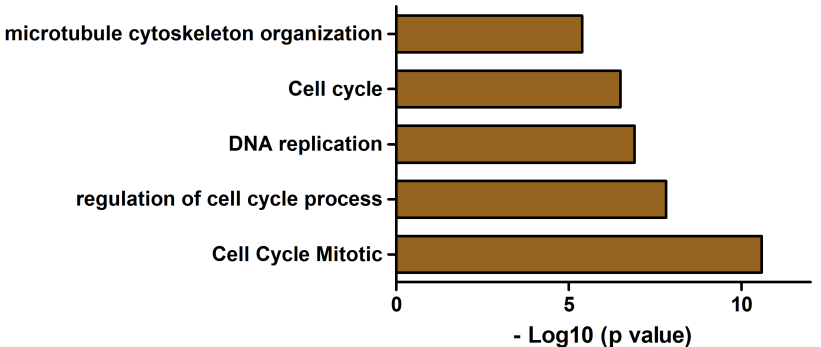

\section{Figure 5}

Analysis of hub genes and modules in the overlap DEGs. (A) PPI network of overlapped genes constructed using Cytoscape. Sizes of dots are proportional to the score and gray edges indicate protein interactions. (B) Scatter plot view of centrality degree. X-axis represents centrality outdegree and y-axis represents centrality indegree. (C) PPI network of hub overlapped genes (centrality degree $\geq 5$ ), constructed by Cytoscape. (D) Scatter plot view of hub overlapped genes (centrality degree $\geq 5$ ), which all were down-regulated. (E) Enrichment analysis of hub overlapped genes, produced by Metascape. 

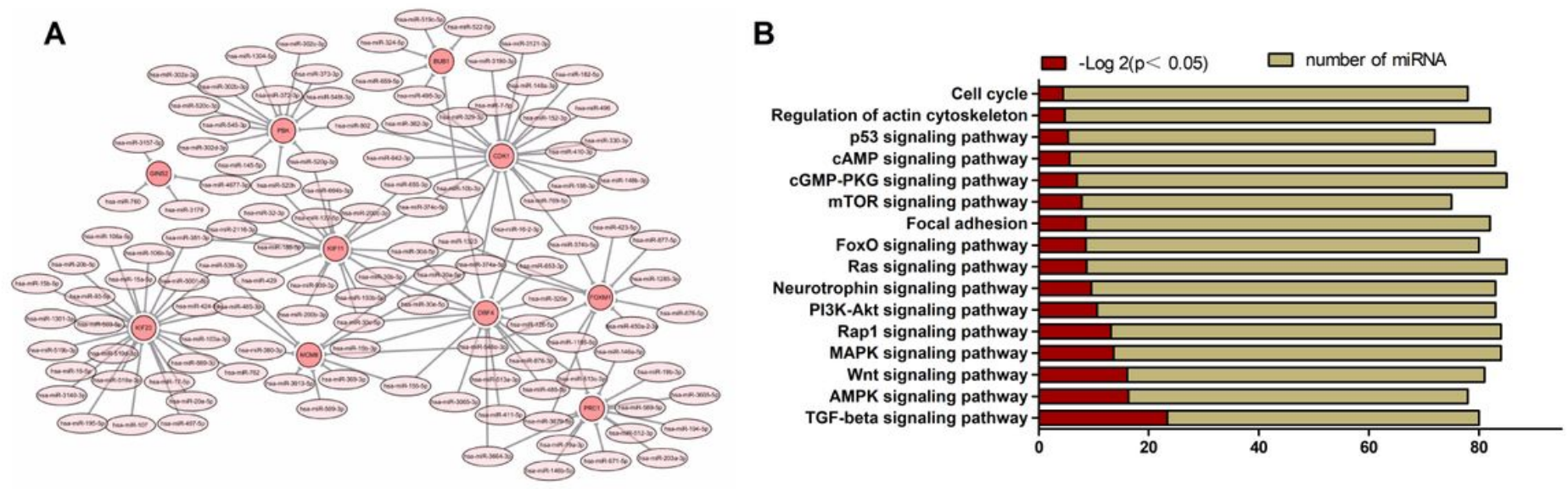

\section{Figure 6}

MiRNA-mRNA regulatory networks in hub overlapped genes. (A) MiRNA-mRNA (CDK1, KIF11, KIF23, BUB1, MAD2L1, KIAA0101, PBK, GINS2, PRC1, FOXM1, DBF4 and MCM8) regulatory networks. The red node represents the gene from the subnetwork, and the purple node represents the miRNA; The red edge indicates the interaction of mRNAs-mRNAs, and the blue edge indicates the interaction of miRNAsmRNAs. (B) Bar graph for KEGG pathway enrichment analysis of predicted miRNAs (CDK1, KIF11, KIF23, BUB1, MAD2L1, KIAA0101, PBK and GINS2). 